\title{
Synthesis of fluoranthenes by hydroarylation of alkynes catalyzed by gold(I) or gallium trichloride
}

\author{
Sergio Pascual ${ }^{1}$, Christophe Bour ${ }^{1}$, Paula de Mendoza ${ }^{1}$ \\ and Antonio M. Echavarren ${ }^{* 1,2}$
}

\author{
Full Research Paper \\ Address: \\ ${ }^{1}$ Institute of Chemical Research of Catalonia (ICIQ), Av. Països \\ Catalans 16, 43007 Tarragona, Spain and ${ }^{2}$ Additional affiliation: \\ Departament de Química Analítica i Química Orgànica, Universitat \\ Rovira i Virgili, C/ Marcel-li Domingo s/n, 43007 Tarragona, Spain \\ Email: \\ Antonio M. Echavarren* - aechavarren@iciq.es \\ * Corresponding author \\ Keywords: \\ alkynes; gold(I) catalysis; hydroarylation; polyarenes
}

Open Access

\author{
Beilstein J. Org. Chem. 2011, 7, 1520-1525. \\ doi:10.3762/bjoc.7.178 \\ Received: 08 September 2011 \\ Accepted: 28 October 2011 \\ Published: 14 November 2011 \\ This article is part of the Thematic Series "Gold catalysis for organic \\ synthesis". \\ Guest Editor: F. D. Toste \\ (C) 2011 Pascual et al; licensee Beilstein-Institut.
}

License and terms: see end of document.

\begin{abstract}
Electrophilic gold(I) catalyst 6 competes with $\mathrm{GaCl}_{3}$ as the catalyst of choice in the synthesis of fluoranthenes by intramolecular hydroarylation of alkynes. The potential of this catalyst for the preparation of polyarenes is illustrated by a synthesis of two functionalized decacyclenes in a one-pot transformation in which three $\mathrm{C}-\mathrm{C}$ bonds are formed with high efficiency.
\end{abstract}

\section{Introduction}

Electrophilic activation of alkynes in functionalized substrates by gold catalysts allows for the synthesis of complex molecules under mild conditions [1-8]. Alkynes can react in goldcatalyzed Friedel-Crafts-type reactions with arenes to give products resulting from the intermolecular hydroarylation of the alkynes (or alkenylation of the arenes) [9-21]. In addition to gold, the intramolecular version of this reaction was also carried out with $\mathrm{Ru}(\mathrm{II})$ [22], Pt(II) [12,22,23], Pt(IV) [24], Ga(III) $[25,26]$, and $\operatorname{Hg}(\mathrm{II})[27,28]$ as catalysts.

Electron-rich indoles also react with alkynes in the presence of gold catalysts to form 6-8-membered rings [29-31]. A similar reaction can also be carried out with $\mathrm{GaCl}_{3}$ [32] and $\mathrm{Pt}(\mathrm{II})$ [33] as catalysts. In contrast, alkynyl furans react with gold to give phenols by using $\mathrm{Au}(\mathrm{III}), \mathrm{Au}(\mathrm{I})$ [1,2,34-37], or Pt(II) as the catalyst [38,39].

In our efforts towards the synthesis of large polyarenes [40-43], which are related to the fullerenes [44], we used the palladiumcatalyzed arylation reaction as the main tool [45-48]. We decided to try the triple hydroarylation of substrates of type $\mathbf{1}$ to give 3,9,15-triaryldiacenaphtho[1,2-j:1',2'-l]fluoranthenes 2 with $\mathrm{X}$ and $\mathrm{Y}$ substitutes at strategic positions, which could be activated by palladium in subsequent intramolecular arylations 


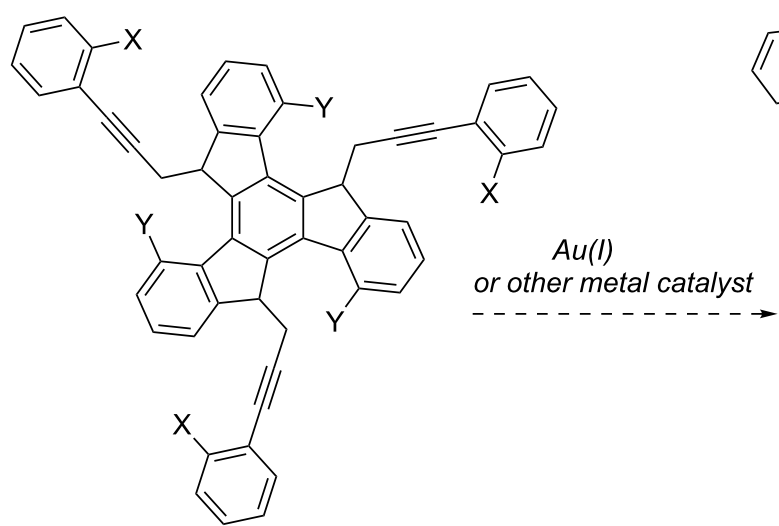

1: $\mathrm{X}, \mathrm{Y}=\mathrm{Br}, \mathrm{OTf}$

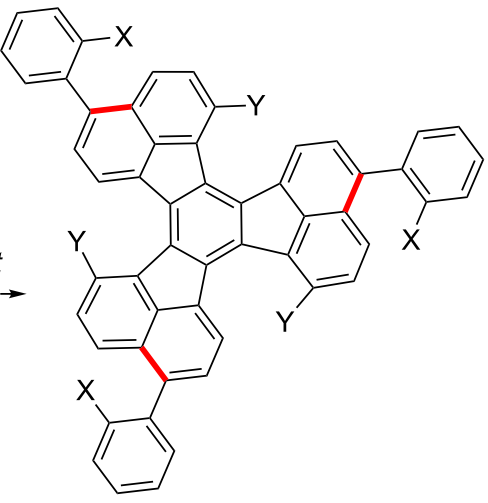

2

Scheme 1: Proposed metal catalyzed annulation for the synthesis of triaryldiacenaphtho[1,2-j:1',2'-/]fluoranthenes 2.

(Scheme 1). Substituted fluoranthenes are of interest since some derivatives have been shown to be useful in light-emitting devices [49-52]. Fluoranthene derivatives have already been synthesized by palladium-catalyzed arylation reactions [53,54]. Strategically halogenated decacyclenes with a substitution pattern similar to that of $\mathbf{2}$ have been used for the synthesis of circumtrindene by flash vacuum pyrolysis [55]. Here we report the results on the synthesis of large polyarenes $\mathbf{2}$ and more simple 3-arylfluoranthenes by using gold(I)- or gallium(III)catalyzed hydroarylation reactions.

\section{Results and Discussion}

First, we examined the cyclization of 3 to give 4 or $4^{\prime}$ [22,24,26] (Table 1) with cationic gold(I) catalysts 5 [56] and 6 [57] (Figure 1), which have been demonstrated to be amongst the best catalysts in many gold(I)-catalyzed cyclizations [6,58]. No reaction was observed with complex 5 after heating for 5 min at $70{ }^{\circ} \mathrm{C}$ in $\mathrm{CH}_{2} \mathrm{Cl}_{2}$ under microwave irradiation (Table 1, entry 1 ), whereas the more electrophilic $\mathbf{6}$, bearing a less donating phosphite ligand, led almost quantitatively to $\mathbf{4}^{\prime}$ (Table 1, entry 2). Under these conditions, $\mathrm{AuCl}_{3}$ was not effective as a catalyst (Table 1, entry 3 ). As previously reported $[25,26], \mathrm{GaCl}_{3}$ is an excellent catalyst for the cyclization of $\mathbf{3}$ to give 4' (Table 1, entry 4). In all cases the reaction proceeds exclusively though the 6-exo-dig pathway.

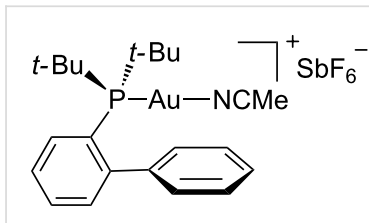

5

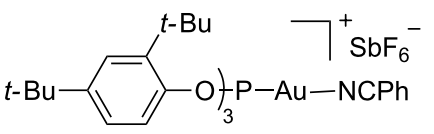

6
Figure 1: Cationic gold complexes $\mathbf{5}$ and $\mathbf{6}$.

The cyclization of 9-(3-phenylprop-2-ynyl)-9H-fluorene (7a) to form 3-phenylfluoranthene (8a) [59] was also examined by using catalysts $\mathbf{5}, \mathbf{6}$, and $\mathrm{GaCl}_{3}$ (Table 2). Since the initial

Table 1: Hydroarylation of 3 to give dihydronaphthalene $4^{\prime}$ a $^{a}$
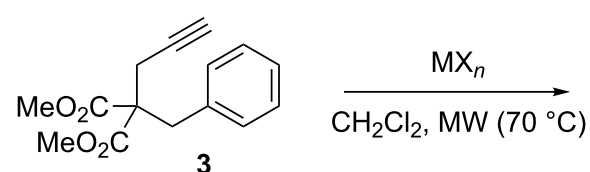<smiles>C=C1CC(C(C)=O)(C(=O)OC)Cc2ccccc21</smiles>

4<smiles>COC(=O)C1(C(O)OC)C=C(C)c2ccccc2C1</smiles>

\begin{tabular}{lll}
\hline entry & $\mathrm{MX}_{n}$ & 4' (yield, \%) \\
\hline 1 & $\mathbf{5}$ & - $^{\mathrm{b}}$ \\
2 & $\mathbf{6}$ & 99 \\
3 & $\mathrm{AuCl}_{3}$ & $\mathbf{C}^{\mathrm{c}}$ \\
4 & $\mathrm{GaCl}_{3}$ & 99 \\
\hline
\end{tabular}

a 2 mol \% catalyst, microwave irradiation, 5 min. ${ }^{b} 100 \% 3$ was recovered. ${ }^{c} 87 \% 3$ was recovered. 
Table 2: Hydroarylation of 9-(3-phenylprop-2-ynyl)-9H-fluorene (7a) to give 3-phenylfluoranthene (8a). ${ }^{a}$

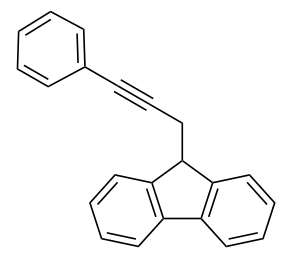

$7 a$

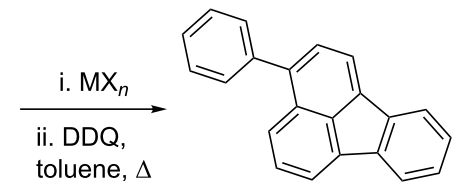

$8 a$

\begin{tabular}{|c|c|c|c|c|c|}
\hline entry & $\mathrm{MX}_{n}(\mathrm{~mol} \%)$ & solvent & $T\left({ }^{\circ} \mathrm{C}\right)$ & $t(\mathrm{~h})$ & yield (\%) \\
\hline 1 & $5(2)$ & $\mathrm{CH}_{2} \mathrm{Cl}_{2}$ & $70^{\mathrm{b}}$ & 0.7 & $-^{c}$ \\
\hline 2 & $5(5)$ & toluene & 110 & 1 & $-d$ \\
\hline 3 & $6(5)$ & $\mathrm{CH}_{2} \mathrm{Cl}_{2}$ & r.t. & 17 & 64 \\
\hline 4 & $6(2)$ & $\mathrm{CH}_{2} \mathrm{Cl}_{2}$ & r.t. & 16 & 70 \\
\hline 5 & $6(1)$ & $\mathrm{CH}_{2} \mathrm{Cl}_{2}$ & r.t. & 16 & 70 \\
\hline 6 & $\mathrm{PtCl}_{2}(5)$ & toluene & 110 & 17 & $-^{c}$ \\
\hline 7 & $\mathrm{AuCl}_{3}(5)$ & toluene & 110 & 17 & $-^{c}$ \\
\hline 8 & $\mathrm{InCl}_{3}(5)$ & toluene & 110 & 17 & $-^{d}$ \\
\hline 9 & $\mathrm{GaCl}_{3}(2)$ & $\mathrm{CH}_{2} \mathrm{Cl}_{2}$ & r.t. & 26 & $16^{e}$ \\
\hline 10 & $\mathrm{GaCl}_{3}(2)$ & toluene & $70^{b}$ & 0.2 & 57 \\
\hline 11 & $\mathrm{FeCl}_{3} \cdot 6 \mathrm{H}_{2} \mathrm{O}(10)$ & $D_{C E}^{f}$ & r.t. & 40 & $36^{e}$ \\
\hline 12 & $\mathrm{FeCl}_{3} \cdot 6 \mathrm{H}_{2} \mathrm{O}(5)$ & $D_{C E}^{f}$ & $70^{\mathrm{b}}$ & 0.2 & $34^{e}$ \\
\hline
\end{tabular}

${ }^{a}$ Crude reaction mixtures were aromatized by heating in toluene with DDQ (3 equiv) for $12 \mathrm{~h}$. ${ }^{\mathrm{b}}$ Microwave irradiation. ${ }^{\mathrm{c}}$ No reaction. ${ }^{\mathrm{d}}$ Product decomposition. ${ }^{e}$ Yield determined by ${ }^{1} \mathrm{H}$ NMR. ${ }^{\mathrm{f}} \mathrm{DCE}=1$,2-dichloroethane.

gold(I)-catalyzed reaction provided a mixture of 3-phenyl1,10b-dihydrofluoranthene, 3-phenyl-1,2,3,10b-tetrahydrofluoranthene, and $\mathbf{8 a}$, the crude mixtures were treated with excess DDQ in toluene under reflux to provide pure 8a. No reaction or decomposition was observed when the reaction was carried out with gold(I) complex 5 (Table 2, entries 1 and 2). In contrast, the more electrophilic gold(I) complex 6 with phosphite as the ligand led to $\mathbf{8 a}$ in $64-70 \%$ yield by stirring at room temperature in $\mathrm{CH}_{2} \mathrm{Cl}_{2}$ (Table 2, entries 3-5). Satisfactory results were obtained by simply using $1 \mathrm{~mol} \%$ of 6 (Table 2 , entry 5 ). No reaction was observed with $\mathrm{PtCl}_{2}$ or $\mathrm{AuCl}_{3}$ even after heating in toluene under reflux (Table 2, entries 3-5). Whereas $\mathrm{InCl}_{3}$ led to decomposition of $\mathbf{7 a}$ under these conditions (Table 2, entry 6), $\mathrm{GaCl}_{3}$ led to $\mathbf{8 a}$, although satisfactory results were only obtained in toluene at $70{ }^{\circ} \mathrm{C}$ (Table 1 , entry 10). Interestingly, $\mathrm{FeCl}_{3}$ was also catalytically active, although fluoranthene 8a was only obtained in moderate yields (Table 2, entries 11 and 12). The reaction of $\mathbf{3 a}$ with $\mathrm{Pd}(\mathrm{OAc})_{2}$ as catalyst proceeded differently to give known (E)-9-(3-phenylallylidene)$9 \mathrm{H}$-fluorene (9) [60], presumably via the formation of the corresponding allene as an intermediate (Scheme 2).

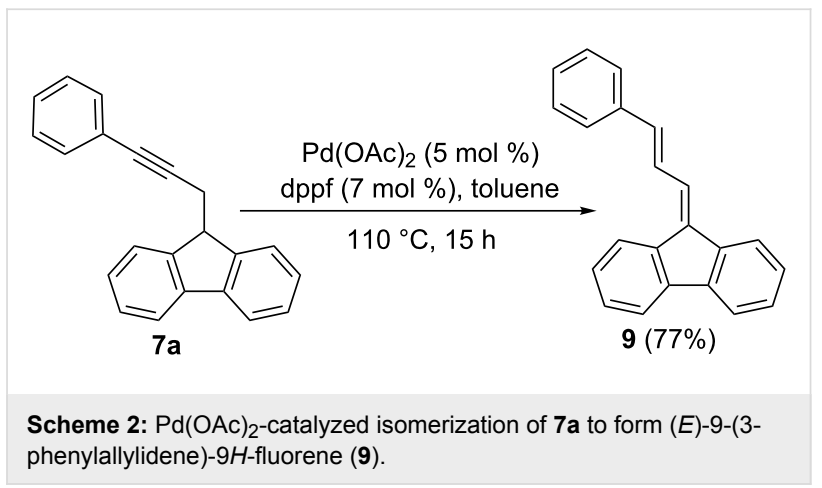

Substrates $\mathbf{7 b}-\mathbf{j}$, prepared by alkylation of fluorenyl lithium with the corresponding propargyl bromide or by Sonogashira couplings of 9-(prop-2-ynyl)-9H-fluorene [61], were cyclized by using gold(I) complex 6 or $\mathrm{GaCl}_{3}$ as the catalyst (Table 3). Although both catalysts could be used for the synthesis of 3 -arylfluoranthenes $\mathbf{8 b}-\mathbf{h}$, better yields were obtained with $\mathrm{GaCl}_{3}$ in toluene at $100{ }^{\circ} \mathrm{C}$. However, in the case of 9-(3bromoprop-2-yn-1-yl)-9H-fluorene (7i), gold(I) complex 6 gave more satisfactory results (Table 3 , compare entries 10 and 11). The reaction proceeded satisfactorily with aryl-substituted substrates bearing either electron-donating ( $p$-Me, $o$-OMe) or electron-withdrawing ( $p-\mathrm{Cl}, p-\mathrm{Br}, p-\mathrm{CN}, p-\mathrm{NO}_{2}$ ) groups. However, no reaction was observed for $n$-butyl derivative $\mathbf{7 j}$ with 6 or with $\mathrm{GaCl}_{3}$ (Table 3, entries 12 and 13).

Cyclization of substrate $\mathbf{7 k}$, having an electron-rich aryl group at the alkyne, with catalyst 6 gave 1,10b-dihydrofluoranthene 9 cleanly in quantitative yield (Scheme 3).

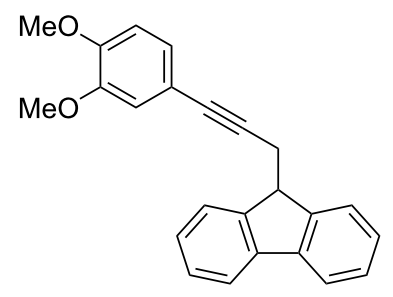

$7 \mathrm{k}$

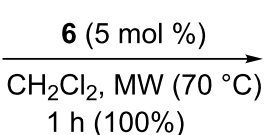

$1 \mathrm{~h}(100 \%)$

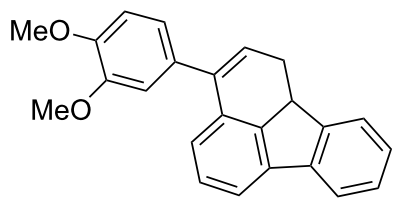


Table 3: Hydroarylation of $7 \mathbf{b}-\mathbf{j}$ to give 3-substituted fluoranthenes $8 \mathbf{b}-\mathbf{i}^{{ }^{a}}$

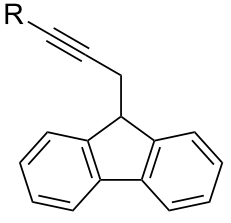

$7 b-j$

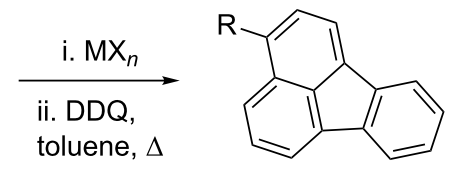

$8 b-j$

\begin{tabular}{|c|c|c|c|c|c|c|c|}
\hline entry & fluorene & $\mathrm{R}$ & $\mathrm{MX}_{n}(\mathrm{~mol} \%)$ & solvent & $T\left({ }^{\circ} \mathrm{C}\right)$ & $t(\mathrm{~h})$ & yield (\%) \\
\hline 1 & $7 b$ & $p$-Tol & $\mathrm{GaCl}_{3}(5)$ & toluene & $100^{b}$ & 0.2 & 45 \\
\hline 2 & $7 b$ & p-Tol & $6(5)$ & $\mathrm{CH}_{2} \mathrm{Cl}_{2}$ & r.t. & 17 & 28 \\
\hline 3 & $7 c$ & $p-\mathrm{ClC}_{6} \mathrm{H}_{4}$ & $\mathrm{GaCl}_{3}(5)$ & toluene & $100^{b}$ & 0.2 & 71 \\
\hline 4 & $7 d$ & $p-\mathrm{NCC}_{6} \mathrm{H}_{4}$ & $\mathrm{GaCl}_{3}(2)$ & toluene & $100^{b}$ & 0.2 & 88 \\
\hline 5 & $7 e$ & $p-\mathrm{O}_{2} \mathrm{NC}_{6} \mathrm{H}_{4}$ & $\mathrm{GaCl}_{3}(2)$ & toluene & $70^{\mathrm{b}}$ & 0.2 & 92 \\
\hline 6 & $7 f$ & $o-\mathrm{MeOC}_{6} \mathrm{H}_{4}$ & $6(5)$ & $\mathrm{CH}_{2} \mathrm{Cl}_{2}$ & r.t. & 17 & 17 \\
\hline 7 & $7 f$ & $o-\mathrm{MeOC}_{6} \mathrm{H}_{4}$ & $\mathrm{GaCl}_{3}(5)$ & toluene & $100^{\mathrm{b}}$ & 0.2 & 57 \\
\hline 8 & $7 g$ & $o-\mathrm{BrC}_{6} \mathrm{H}_{4}$ & $\mathrm{GaCl}_{3}(5)$ & toluene & $100^{b}$ & 0.2 & 44 \\
\hline 9 & $7 \mathrm{~h}$ & $\mathrm{C}_{6} \mathrm{~F}_{5}$ & $\mathrm{GaCl}_{3}(5)$ & toluene & $100^{b}$ & 2 & 74 \\
\hline 10 & $7 \mathbf{i}$ & $\mathrm{Br}$ & $6(5)$ & $\mathrm{CH}_{2} \mathrm{Cl}_{2}$ & r.t. & 20 & 44 \\
\hline 11 & $7 \mathbf{i}$ & $\mathrm{Br}$ & $\mathrm{GaCl}_{3}(5)$ & toluene & $100^{b}$ & 0.2 & 21 \\
\hline 12 & $7 \mathbf{j}$ & $n-\mathrm{Bu}$ & $6(5)$ & $\mathrm{CH}_{2} \mathrm{Cl}_{2}$ & r.t. & 7 & $-^{c}$ \\
\hline 13 & $7 \mathbf{j}$ & $n-\mathrm{Bu}$ & $\mathrm{GaCl}_{3}(2)$ & toluene & $70^{\mathrm{b}}$ & 0.2 & $-^{c}$ \\
\hline
\end{tabular}

${ }^{a}$ Crude reaction mixtures were aromatized by heating in toluene with DDQ (3 equiv) for $12 \mathrm{~h}$. ${ }^{\mathrm{b}}$ Microwave irradiation. ${ }^{\mathrm{C}}$ No reaction.

Derivatives 1a and $\mathbf{1 b}$ were readily prepared by the triple alkylation of the lithium anion of 4,9,14-trimethoxytruxene (Scheme 4) [41,62]. The cyclization reaction was carried out efficiently with gold(I) catalyst $6(15 \mathrm{~mol} \%)$ at room temperature in $\mathrm{CH}_{2} \mathrm{Cl}_{2}$ to give triaryl substituted diacenaphtho[1,2$\left.j: 1^{\prime}, 2^{\prime}-1\right]$ fluoranthenes (decacyclenes) $\mathbf{2 a}$ and $\mathbf{2 b}$ in very good overall yields after aromatization of the crude products with DDQ. Remarkably, this triple hydroarylation occurs efficiently with an average yield per $\mathrm{C}-\mathrm{C}$ bond formation that is greater than $90 \%$.

\section{Conclusion}

Highly electrophilic gold(I) catalyst 6 with a bulky phosphite ligand competes with $\mathrm{GaCl}_{3}$ as the catalyst of choice for the hydroarylation of alkynes. The synthetic potential of this catalyst is illustrated by the synthesis of functionalized triarylated

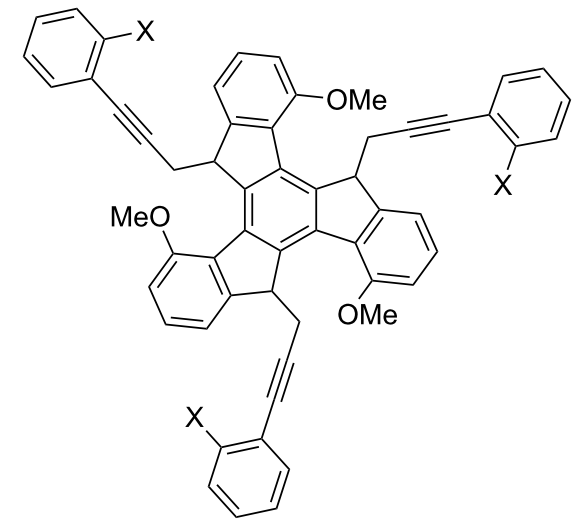

1a: $X=\mathrm{H}$

1b: $X=\mathrm{Br}$

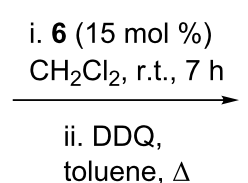

toluene, $\Delta$

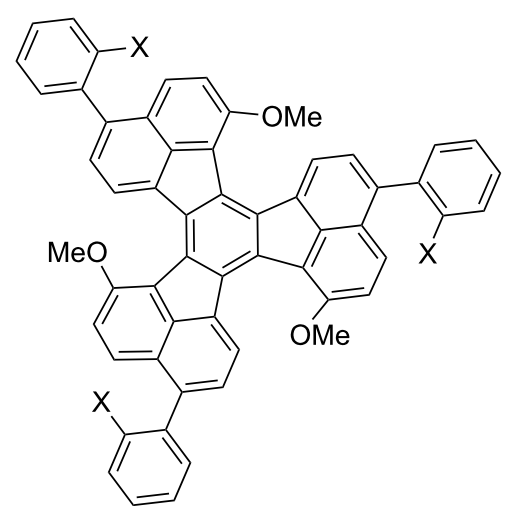

2a: $X=\mathrm{H}(72 \%)$

2b: $X=\operatorname{Br}(77 \%)$ 
decacyclenes in which three $\mathrm{C}-\mathrm{C}$ bonds are formed with high efficiency in a one-pot transformation. The reaction is totally compatible with aryl bromides, which do not undergo subsequent arylation reaction due to the inertness of gold(I) catalysts towards oxidative addition reactions under homogeneous conditions $[63,64]$.

\section{Supporting Information}

Supporting Information features experimental details and characterization data for new compounds.

\section{Supporting Information File 1}

Experimental details

[http://www.beilstein-journals.org/bjoc/content/

supplementary/1860-5397-7-178-S1.pdf]

\section{Acknowledgements}

This work was supported by the (MICINN (CTQ2010-16088/ BQU and Consolider Ingenio 2010, Grant CSD2006-0003), the AGAUR (2009SGR47), and by the European Commission within the projects PicoInside (Contract No. IST-015847) and AtMol (Contract No. ICT-270028).

\section{References}

1. Hashmi, A. S. K.; Hutchings, G. J. Angew. Chem., Int. Ed. 2006, 45, 7896-7936. doi:10.1002/anie.200602454

2. Hashmi, A. S. K. Chem. Rev. 2007, 107, 3180-3211. doi:10.1021/cr000436x

3. Zhang, L.; Sun, J.; Kozmin, S. A. Adv. Synth. Catal. 2006, 348, 2271-2296. doi:10.1002/adsc.200600368

4. Fürstner, A.; Davies, P. W. Angew. Chem., Int. Ed. 2007, 46, 3410-3449. doi:10.1002/anie.200604335

5. Gorin, D. J.; Toste, F. D. Nature 2007, 446, 395-403. doi:10.1038/nature05592

6. Jiménez-Núñez, E.; Echavarren, A. M. Chem. Rev. 2008, 108, 3326-3350. doi:10.1021/cr0684319

7. Gorin, D. J.; Sherry, B. D.; Toste, F. D. Chem. Rev. 2008, 108, 3351-3378. doi:10.1021/cr068430g

8. Michelet, V.; Toullec, P. Y.; Genêt, J. P. Angew. Chem., Int. Ed. 2008, 47, 4268-4315. doi:10.1002/anie.200701589

9. Nevado, C.; Echavarren, A. M. Synthesis 2005, 167-182. doi:10.1055/s-2005-861781

10. de Mendoza, P.; Echavarren, A. M. Pure Appl. Chem. 2010, 82, 801-820. doi:10.1351/PAC-CON-09-10-06

11. Reetz, M. T.; Sommer, K. Eur. J. Org. Chem. 2003, 3485-3496. doi:10.1002/ejoc.200300260

12. Shi, Z.; He, C. J. Org. Chem. 2004, 69, 3669-3671. doi:10.1021/jo0497353

13. Li, Z.; Shi, Z.; He, C. J. Organomet. Chem. 2005, 690, 5049-5054. doi:10.1016/j.jorganchem.2005.03.009

14. Nevado, C.; Echavarren, A. M. Chem.-Eur. J. 2005, 11, 3155-3164. doi:10.1002/chem.200401069
15. Dankwardt, J. W. Tetrahedron Lett. 2001, 42, 5809-5812. doi:10.1016/S0040-4039(01)01146-7

16. Fürstner, A.; Mamane, V. J. Org. Chem. 2002, 67, 6264-6267. doi:10.1021/jo025962y

17. Mamane, V.; Hannen, P.; Fürstner, A. Chem.-Eur. J. 2004, 10, 4556-4575. doi:10.1002/chem.200400220

18. Soriano, E.; Marco-Contelles, J. Organometallics 2006, 25 , 4542-4553. doi:10.1021/om0605332

19. Seregin, I. V.; Gevorgyan, V. J. Am. Chem. Soc. 2006, 128, 12050-12051. doi:10.1021/ja063278|

20. Menon, R. S.; Findlay, A. D.; Bissember, A. C.; Banwell, M. G. J. Org. Chem. 2009, 74, 8901-8903. doi:10.1021/jo902032p

21. Jurberg, I. D.; Gagosz, F. J. Organomet. Chem. 2011, 696, 37-41. doi:10.1016/j.jorganchem.2010.06.017

22. Chatani, N.; Inoue, H.; Ikeda, T.; Murai, S. J. Org. Chem. 2000, 65, 4913-4918. doi:10.1021/jo000255v

23. Fürstner, A.; Kennedy, J. W. J. Chem.-Eur. J. 2006, 12, 7398-7410. doi:10.1002/chem.200600592

24. Pastine, S. J.; Youn, S. W.; Sames, D. Org. Lett. 2003, 5, 1055-1058. doi:10.1021/ol034177k

25. Inoue, H.; Chatani, N.; Murai, S. J. Org. Chem. 2002, 67, 1414-1417. doi:10.1021/jo016232d

26. Li, H. J.; Guillot, R.; Gandon, V. J. Org. Chem. 2010, 75, 8435-8449. doi:10.1021/jo101709n

27. Nishizawa, M.; Takao, H.; Yadav, V. K.; Imagawa, H.; Sugihara, T. Org. Lett. 2003, 5, 4563-4565. doi:10.1021/ol035622e

28. Nishizawa, M.; Imagawa, H.; Yamamoto, H. Org. Biomol. Chem. 2010, 8, 511-521. doi:10.1039/b920434b

29. Ferrer, C.; Echavarren, A. M. Angew. Chem., Int. Ed. 2006, 45, 1105-1109. doi:10.1002/anie.200503484

30. Ferrer, C.; Amijs, C. H. M.; Echavarren, A. M. Chem.-Eur. J. 2007, 13, 1358-1373. doi:10.1002/chem.200601324

31. Ferrer, C.; Escribano-Cuesta, A.; Echavarren, A. M. Tetrahedron 2009, 65, 9015-9020. doi:10.1016/j.tet.2009.08.067

32. Yadav, J. S.; Reddy, B. V. S.; Padmavani, B.; Gupta, M. K. Tetrahedron Lett. 2004, 45, 7577-7579. doi:10.1016/j.tetlet.2004.08.126

33. Bhuvaneswari, S.; Jeganmohan, M.; Cheng, C.-H. Chem.-Eur. J. 2007, 13, 8285-8293. doi:10.1002/chem.200700589

34. Rudolph, M.; McCreery, M. Q.; Frey, W.; Hashmi, A. S. K. Beilstein J. Org. Chem. 2011, 7, 794-801. doi:10.3762/bjoc.7.90

35. Hashmi, A. S. K.; Blanco, M. C. Eur. J. Org. Chem. 2006, 4340-4342. doi:10.1002/ejoc.200600546

36. Hashmi, A. S. K.; Haufe, P.; Schmid, C.; Nass, A. R.; Frey, W. Chem.-Eur. J. 2006, 12, 5376-5382. doi:10.1002/chem.200600192

37. Hashmi, A. S. K.; Yang, M. S. W.; Rominger, F. Angew. Chem., Int. Ed. 2011, 50, 5762-5765. doi:10.1002/anie.201100989

38. Martín-Matute, B.; Cárdenas, D. J.; Echavarren, A. M. Angew. Chem., Int. Ed. 2001, 40, 4754-4757. doi:10.1002/1521-3773(20011217)40:24<4754::AID-ANIE4754>3.0.CO ;2-9

39. Martín-Matute, B.; Nevado, C.; Cárdenas, D. J.; Echavarren, A. M. J. Am. Chem. Soc. 2003, 125, 5757-5766. doi:10.1021/ja029125p

40. Gómez-Lor, B.; de Frutos, Ó.; Echavarren, A. M. Chem. Commun. 1999, 2431-2432. doi:10.1039/a906990i

41. Gómez-Lor, B.; González-Cantalapiedra, E.; Ruiz, M.; de Frutos, Ó.; Cárdenas, D. J.; Santos, A.; Echavarren, A. M. Chem.-Eur. J. 2004, 10, 2601-2608. doi:10.1002/chem.200306023

42. Echavarren, A. M.; Gómez-Lor, B.; González, J. J.; de Frutos, Ó. Synlett 2003, 585-597. doi:10.1055/s-2003-38382 
43. Pascual, S.; de Mendoza, P.; Echavarren, A. M. Org. Biomol. Chem. 2007, 5, 2727-2734. doi:10.1039/b707940k

44. Otero, G.; Biddau, G.; Sánchez-Sánchez, C.; Caillard, R.; López, M. F.; Rogero, C.; Palomares, F. J.; Cabello, N.; Basanta, M. A.; Ortega, J.; Méndez, J.; Echavarren, A. M.; Pérez, R.; Gómez-Lor, B.; Martín-Gago, J. A. Nature 2008, 454, 865-868. doi:10.1038/nature07193

45. García-Cuadrado, D.; Braga, A. A. C.; Maseras, F.; Echavarren, A. M. J. Am. Chem. Soc. 2006, 128, 1066-1067. doi:10.1021/ja056165v

46. García-Cuadrado, D.; de Mendoza, P.; Braga, A. A. C.; Maseras, F.; Echavarren, A. M. J. Am. Chem. Soc. 2007, 129, 6880-6886. doi:10.1021/ja071034a

47. Pascual, S.; de Mendoza, P.; Braga, A. A. C.; Maseras, F.; Echavarren, A. M. Tetrahedron 2008, 64, 6021-6029. doi:10.1016/j.tet.2008.01.056

48. Livendahl, M.; Echavarren, A. M. Isr. J. Chem. 2010, 50, 630-651. doi:10.1002/ijch.201000040

49. Kim, S.-K.; Park, J.-W. J. Nanosci. Nanotechnol. 2008, 8, 4787-4792. doi:10.1166/jnn.2008.IC69

50. Kim, S.-K.; Jaung, J.-Y.; Park, J.-W. Mol. Cryst. Liq. Cryst. 2009, 498, 140-150. doi:10.1080/15421400802615352

51. Yan, Q.; Zhou, Y.; Ni, B.-B.; Ma, Y.; Wang, J.; Pei, J.; Cao, Y. J. Org. Chem. 2008, 73, 5328-5339. doi:10.1021/jo800606b

52. Chiechi, R. C.; Tseng, R. J.; Marchioni, F.; Yang, Y.; Wudl, F. Adv. Mater. 2006, 18, 325-328. doi:10.1002/adma.200501682

53. Wegner, H. A.; Scott, L. T.; de Meijere, A. J. Org. Chem. 2003, 68, 883-887. doi:10.1021/jo020367h

54. Quimby, J. M.; Scout, L. T. Adv. Synth. Catal. 2009, 351, 1009-1013. doi:10.1002/adsc.200900018

55. Ansems, R. B. M.; Scott, L. T. J. Am. Chem. Soc. 2000, 122, 2719-2724. doi:10.1021/ja993028n

56. Nieto-Oberhuber, C.; López, S.; Echavarren, A. M. J. Am. Chem. Soc. 2005, 127, 6178-6179. doi:10.1021/ja042257t

57. Amijs, C. H. M.; López-Carrillo, V.; Raducan, M.; Pérez-Galán, P.; Ferrer, C.; Echavarren, A. M. J. Org. Chem. 2008, 73, 7721-7730. doi:10.1021/jo8014769

58. Raducan, M.; Rodríguez-Escrich, C.; Cambeiro, X. C.; Escudero-Adán, E. C.; Pericàs, M. A.; Echavarren, A. M. Chem. Commun. 2011, 47, 4893-4895. doi:10.1039/c1cc10293a And references therein.

59. Berlman, I. B.; Wirth, H. O.; Steingraber, O. J. J. Am. Chem. Soc. 1968 90, 566-569. doi:10.1021/ja01005a003

60. Kuhn, R.; Winterstein, A. Helv. Chim. Acta 1928, 11, 116-122. doi:10.1002/hlca.19280110108

61. Gautier, J. A.; Miocque, M.; Moskowitz, H. J. Organomet. Chem. 1964, 1, 212-221. doi:10.1016/S0022-328X(00)85489-3

62. Gómez-Lor, B.; de Frutos, Ó.; Ceballos, P. A.; Granier, T.; Echavarren, A. M. Eur. J. Org. Chem. 2001, 2107-2114. doi:10.1002/1099-0690(200106)2001:11<2107::AID-EJOC2107>3.0.C $\mathrm{O} ; 2-\mathrm{F}$

63. Lauterbach, T.; Livendahl, M.; Rosellón, A.; Espinet, P.; Echavarren, A. M. Org. Lett. 2010, 12, 3006-3009. doi:10.1021/ol101012n

64. Livendahl, M.; Espinet, P.; Echavarren, A. M. Platinum Met. Rev. 2011, 55, 212-214. doi:10.1595/147106711X579128

\section{License and Terms}

This is an Open Access article under the terms of the Creative Commons Attribution License

(http://creativecommons.org/licenses/by/2.0), which permits unrestricted use, distribution, and reproduction in any medium, provided the original work is properly cited.

The license is subject to the Beilstein Journal of Organic Chemistry terms and conditions:

(http://www.beilstein-journals.org/bjoc)

The definitive version of this article is the electronic one which can be found at:

doi:10.3762/bjoc. 7.178 